\title{
Expression of human amyloid precursor protein in the skeletal muscles of Drosophila results in age- and activity-dependent muscle weakness
}

\author{
Chul Kim ${ }^{1}$, Sapeckshita Srivastava ${ }^{2}$, Marian Rice ${ }^{3}$, Tanja A Godenschwege ${ }^{4}$, Brooke Bentley $^{5}$, Saranya Ravi ${ }^{3}$, \\ Shuang Shao ${ }^{3}$, Craig T Woodard ${ }^{3}$ and Lawrence M Schwartz ${ }^{1,2,5^{*}}$
}

\begin{abstract}
Background: One of the hallmarks of Alzheimer's disease, and several other degenerative disorders such as Inclusion Body Myositis, is the abnormal accumulation of amyloid precursor protein (APP) and its proteolytic amyloid peptides. To better understand the pathological consequences of inappropriate APP expression on developing tissues, we generated transgenic flies that express wild-type human APP in the skeletal muscles, and then performed anatomical, electrophysiological, and behavioral analysis of the adults.

Results: We observed that neither muscle development nor animal longevity was compromised in these transgenic animals. However, human APP expressing adults developed age-dependent defects in both climbing and flying. We could advance or retard the onset of symptoms by rearing animals in vials with different surface properties, suggesting that human APP expression-mediated behavioral defects are influenced by muscle activity. Muscles from transgenic animals did not display protein aggregates or structural abnormalities at the light or transmission electron microscopic levels. In agreement with genetic studies performed with developing mammalian myoblasts, we observed that co-expression of the ubiquitin E3 ligase Parkin could ameliorate human APP-induced defects.
\end{abstract}

Conclusions: These data suggest that: 1) ectopic expression of human APP in fruit flies leads to age- and activitydependent behavioral defects without overt changes to muscle development or structure; 2) environmental influences can greatly alter the phenotypic consequences of human APP toxicity; and 3) genetic modifiers of APPinduced pathology can be identified and analyzed in this model.

Keywords: amyloid precursor protein (APP) Drosophila, muscle, mitochondria, electron microscopy, apoptosis, Parkin

\section{Background}

Amyloid precursor protein (APP) is a type I glycotransmembrane protein with a large extracellular domain and a short cytoplasmic tail [reviewed in 1]. Its role in normal biological processes is poorly defined, but there is mounting evidence that it plays both autocrine and endocrine roles in neurite growth and enhanced memory function in mice $[2,3]$.

\footnotetext{
* Correspondence: LMS@bio.umass.edu

'Molecular and Cellular Biology Program, University of Massachusetts, Amherst, MA 01003, USA

Full list of author information is available at the end of the article
}

APP became the subject of intense investigation when it was identified as a risk factor for Alzheimer's disease (AD) [4]. Individuals with an extra copy of the APP gene due to trisomy of chromosome 21 (Down Syndrome) also display early onset $\mathrm{AD}$ [5]. One point mutation in APP, referred to as the Swedish mutation, results in an early onset familial AD [6].

APP can be subjected to combinatorial cleavage by three different intramembrane secretases $(\alpha, \beta$, and $\gamma)$ to create a number of smaller peptides [7]. Cleavage by $\alpha$ secretase, known as a non-amyloidogenic pathway, is responsible for a default secretory pathway and predominates in all non-neuronal cells. Cleavage by BACE $(\beta-$
C Biomed Central

() 2011 Kim et al; licensee BioMed Central Ltd. This is an Open Access article distributed under the terms of the Creative Commons Attribution License (http://creativecommons.org/licenses/by/2.0), which permits unrestricted use, distribution, and reproduction in any medium, provided the original work is properly cited. 
site APP cleaving enzyme) represents a minor pathway in most cell types, except for neurons. Like $\alpha$-secretase cleavage of APP, the BACE-mediated fragment of APP undergoes further proteolysis by a $\gamma$-secretase complex to generate small peptides that typically range between 40-44 amino acids, although 46 amino acid fragments can be found in skeletal muscles. APP cleavage products are enriched in the brains of some AD patients [8], and exposure to the $A \beta_{42}$ fragment is highly neurotoxic both in vitro and in in vivo animal models $[9,10]$.

In addition to its well-documented roles in neurodegeneration, APP and its cleaved products have been also implicated in other diseases, most notably sporadic inclusion body myositis (s-IBM) [11], the most common skeletal muscle disorder of the elderly $[12,13]$. It has been reported that muscle biopsies from individuals with s-IBM contain Congo Red inclusions that are immunopositive for both APP [11] and $A \beta_{42}$ [14]. However, this is not a universal observation [15], and proteomic analysis of IBM samples failed to reveal any APP proteolytic products [16]. In fact, a fundamental role for APP in the pathogenesis of s-IBM has been questioned recently $[12,17,18]$.

While the role of APP in s-IBM awaits further analysis of clinical samples, it has been demonstrated that ectopic expression of either APP (either wild-type or Swedish mutant) or $A \beta_{42}$ is sufficient to induce cell death in skeletal muscle either in vitro $[19,20]$ or in vivo [21-23]. The biology of muscle makes it a much more tractable tissue to study than the central nervous system. Thus, studies designed to examine the effects of APP on muscle development and physiology may provide new insights into the general mechanisms that mediate APP induced pathogenesis.

To help define the mechanisms that could mediate APP toxicity in muscle, and to develop a genetic model for testing genetic and environmental interventions that might reduce APP-induced pathogenesis, we generated transgenic flies that express human APP in the skeletal muscles. We found that both genetic and environmental factors can interact to enhance or reduce APP-induced behavioral defects. These data suggest that the transgenic fly may represent a useful tool for defining the molecular mechanisms that mediate APP-induced muscle pathology and for identifying genetic and chemical modulators.

\section{Results and Discussion}

Muscle specific expression of human amyloid precursor protein (hAPP) induces an age-dependent reduction in climbing and flying activity

In order to study the role of human APP (hAPP) on the development and function of skeletal muscles, we took advantage of a transgenic fly line that expresses hAPP under the control of the Upstream Activating Sequence (UAS) [24]. These flies were crossed to a line that expresses the Gal4 transcription factor under the control of the muscle transcription factor Dmef [25], which expresses in all skeletal muscles and a few circadian neurons within the brain [26].

We employed several controls in each of these studies, including nontransgenic $w^{1118}$ flies and transgenic flies expressing bacterial $\beta$-galactosidase (LacZ) under the control of Dmef-Gal4. The expression of LacZ both validated the anticipated expression of hAPP in muscles (data not shown) and controlled any effects caused by the competition of Gal4 promoter for general transcriptional machinery within the cells. Ectopic hAPP expressing flies ( $\boldsymbol{-})$ eclosed in normal numbers and displayed comparable longevity to the control lines $(\mathbf{\Lambda})$ suggesting that ectopic hAPP (-) was non-toxic (Figure 1B).

To assess the effects of ectopic hAPP on behavior, flies were tested for their ability to climb (Figure 1A). Both nontransgenic $\left(w^{1118} \bullet\right)$ and LacZ-expressing (•) flies displayed comparable levels of climbing activity during the one-month testing period. Transgenic flies expressing hAPP exhibited wild-type levels of climbing activity during the first two weeks of adulthood, but it declined during the subsequent weeks (Figure 1C) so that by the end of the fourth week, only about $20 \%$ of the transgenic flies could climb. In contrast, $75 \%$ of 1 month old wild-type $w^{1118}$ control animals could climb. The climbing defect observed in transgenic flies was not due to a loss in their negative-geotropism, but rather, an apparent loss of strength that caused them to fall from the test cylinder before they could reach the test mark.

To evaluate flying behavior, animals were dropped into an oil-coated $500 \mathrm{ml}$ graduated cylinder and then scored for their ability to remain near the top. Approximately $55 \%$ of the hAPP-expressing flies (dark gray) fell to the bottom $200 \mathrm{ml}$ of the cylinder (poor fliers), while only about $12 \%$ of the controls (light gray) fell that distance (Figure 1D). Conversely, about $70 \%$ of the controls (light gray) remained within the top $200 \mathrm{ml}$ (normal fliers), while only $20 \%$ of the transgenic flies (dark gray) were in this category. Taken together, the climbing and flying assays suggest that ectopic expression of hAPP results in an age-dependent defect in motor ability.

\section{Electrophysiological analysis of the neuromuscular junction}

Defects in motor function could reflect aberrations within the central nervous system, at the neuromuscular synapse, or within the muscle itself. To narrow our focus, we performed electrophysiological analyses of control and hAPP-expressing adult Drosophila. Intracellular glass recording microelectrodes were placed in the thoracic dorsal longitudinal flight muscle (DLFM) and 


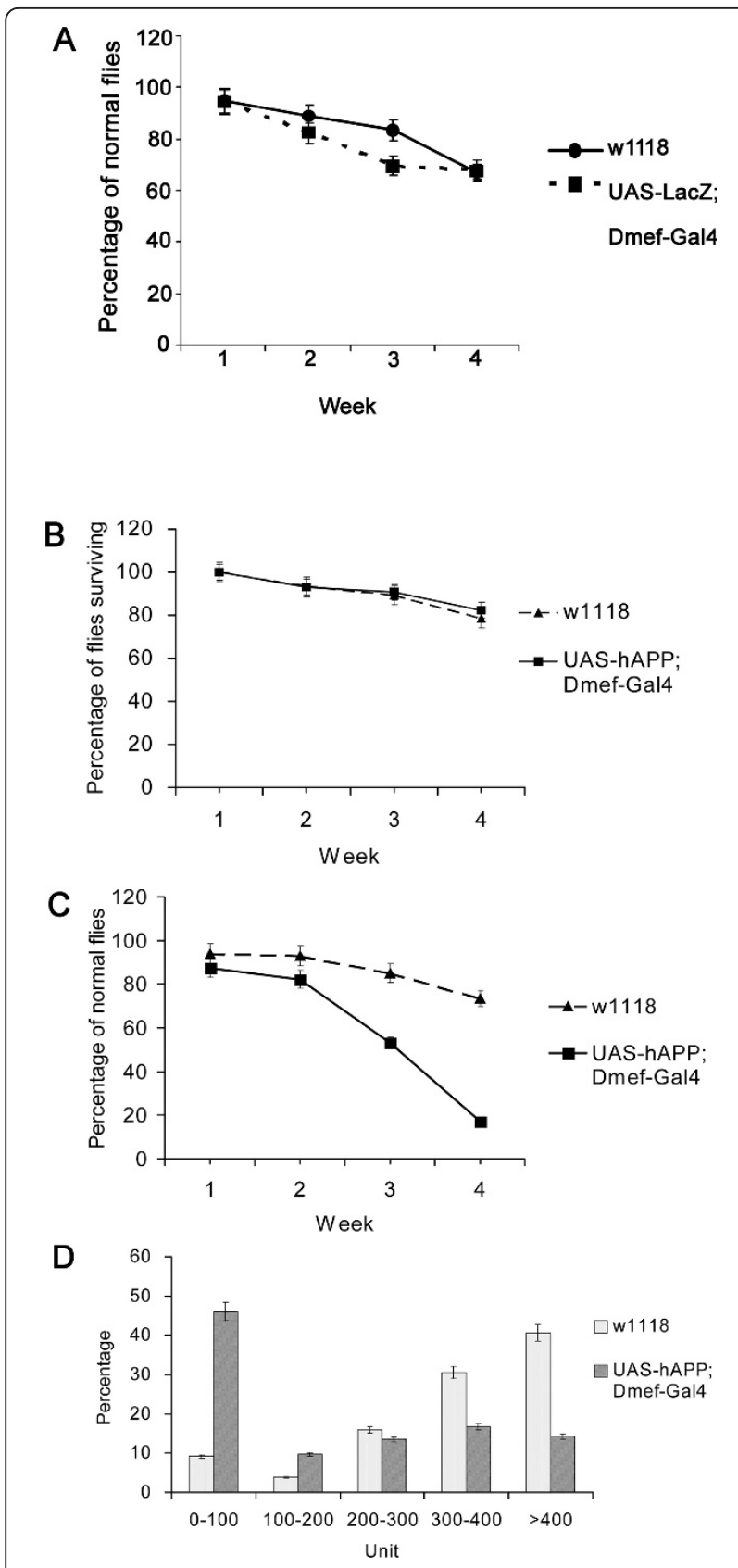

Figure 1 hAPP-expressing flies display age-dependent defects in climbing and flying. A. Climbing assays with wild type and LacZ expressing flies were performed every week. $N=136$ and 85 for $w^{1118}$ and LacZ expressing flies respectively. Mean $+/$ - SEM. B. In order to measure the survival rates of flies, the numbers of $w^{1118}$ and hAPP expressing transgenic flies were counted every week. Initially, 174 wild type and 166 transgenic flies were cultured. Mean +/- SEM. C. Climbing assays with $w^{1118}$ and hAPP expressing transgenic flies in glass vials were conducted every week. $\mathrm{N}=174$ and 166 for wild-type and transgenic animals respectively. Mean $+/$ SEM. D. Flight assays with $w^{1118}$ (light gray) and hAPP expressing flies (dark gray) were performed after five-week incubation in glass vials. Flies between 0-200 ml represent "poor fliers", while animals at $300-500 \mathrm{ml}$ are designated as "good fliers". N = 131 and 90 for wildtype and transgenic animals respectively. Mean +/- SEM. tergotrochanteral motor muscle (TTM) of 1- and 3week old adults, and the motor neurons were stimulated via the giant fibers using tungsten electrodes placed in the brain or directly in the thoracic ganglion. Both $w^{1118}$ control and hAPP-expressing animals displayed normal DFLM muscle responses comprised of an evoked junction potential and a muscle action potential when repeatedly stimulated at $100 \mathrm{~Hz}$ (Figure 2 ). There was no significant difference in amplitude size between animals recorded at 1 week versus animals recorded after 3 weeks (Student T-test, $\mathrm{P}<0.05$, Table 1 ). This suggests that motorneuron activation reliably results in muscle action potentials that should be sufficient to trigger a behavioral outcome mediated by muscle contraction. Consequently, the decay of climbing behavior in 3 week old hAPP expressing flies is not the result of a defect in motorneuron function and thus we focused on potential hAPP effects on the muscle.

\section{Anatomical analysis of muscles}

To evaluate the effects of hAPP on muscle development and function, we performed both light and electron microscopy on the indirect flight muscles of control and hAPP-expressing flies. In Figure 3 we examined coronal ( 3 week old animals) and sagittal (4 week old animals) sections of the thorax to examine both the flight and leg muscles. At the light level, hematoxylin and eosin staining revealed well-developed muscles in both control (3A and $3 \mathrm{C}$ ) and transgenic animals (3B and $3 \mathrm{D}$ ), consistent with their ability to eclose, walk, climb, and fly as adults (Figure 1). Fiber number and diameter were comparable
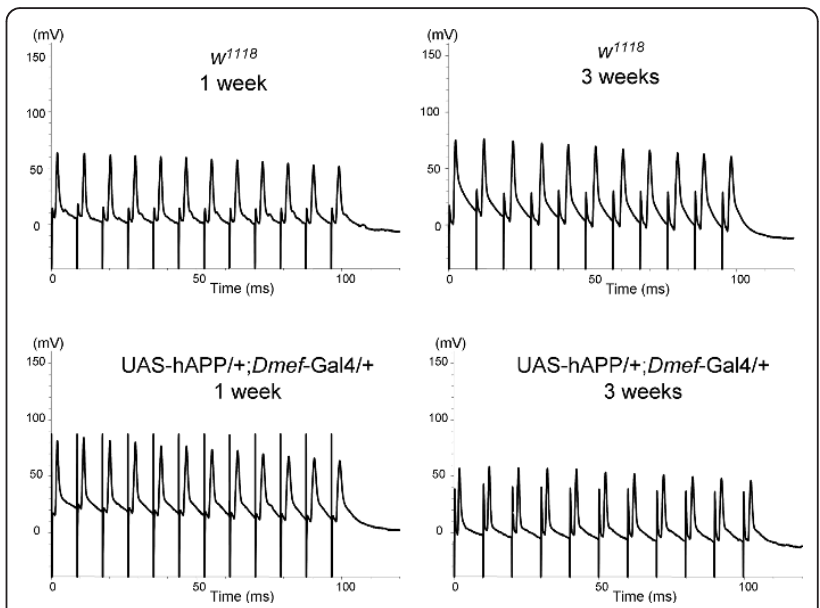

Figure 2 Electrophysiological analysis of wild-type and hAPPexpressing flight muscles. Sample traces of intracellular recordings of muscle responses from the DLFM following electrical stimulation of 1 week (A) and 3 week (B) old wild-type $w^{1118}$ flies and 1 week (C) and 3 week (D) old Dmef-hAPP flies. No obvious differences were detected among the test groups independent of age or genotype. 
Table 1 Amplitude sizes of recordings from the DLFM and the TTM

\begin{tabular}{cccc}
\hline Genotype & N & $\begin{array}{c}\text { DLFM } \\
\text { mV/Stdv/SEM }\end{array}$ & $\begin{array}{c}\text { TTM } \\
\text { mV/Stdv/SEM }\end{array}$ \\
\hline 1 week w $^{1118}$ & 22 & $52.3 / 11.5 / 2.5$ & $25.8 / 8.8 / 1.8$ \\
2 week w $^{1118}$ & 22 & $53.3 / 18.0 / 3.8$ & $23.2 / 7.7 / 1.7$ \\
1 week Dmef/hAPP & 18 & $46.0 / 15.7 / 3.7$ & $26.6 / 7.6 / 1.8$ \\
3 week Dmef/hAPP & 22 & $45.0 / 12.4 / 2.6$ & $23.2 / 6.8 / 2.0$ \\
\hline
\end{tabular}

Intracellular recordings were performed on the DLFM and TTM muscles following electrical stimulation as described in the Materials and Methods. Data are provided for both wild type $w^{1118}$ and Dmef/hAPP flies at both 1 week and 3 weeks. Data is in $\mathrm{mV}$ and presented as the mean/standard deviation/standard error of the mean. $\mathrm{N}$ = number of flies tested for each condition. No significant differences between 1 week and 3 week old flies we observed (Student T-test, $\mathrm{P}<0.05$ )

in wild-type and transgenic animals. These data support the hypothesis that ectopic expression of hAPP does not negatively impact myogenesis.

We also performed transmission electron microscopy on the DLFM from 3-week old wild-type animals, which revealed well-developed Z-lines, M-lines, contractile apparatus and mitochondria (Figure 4A). Neither the muscle fibers themselves nor the internal membrane systems were swollen or disrupted. Anatomically, the DLFM of 3-week old transgenic animals were also grossly normal, and displayed well-defined sarcomeres (Figure 4B). As in the control muscles, dense rows of mitochondria were sandwiched between the bundles of

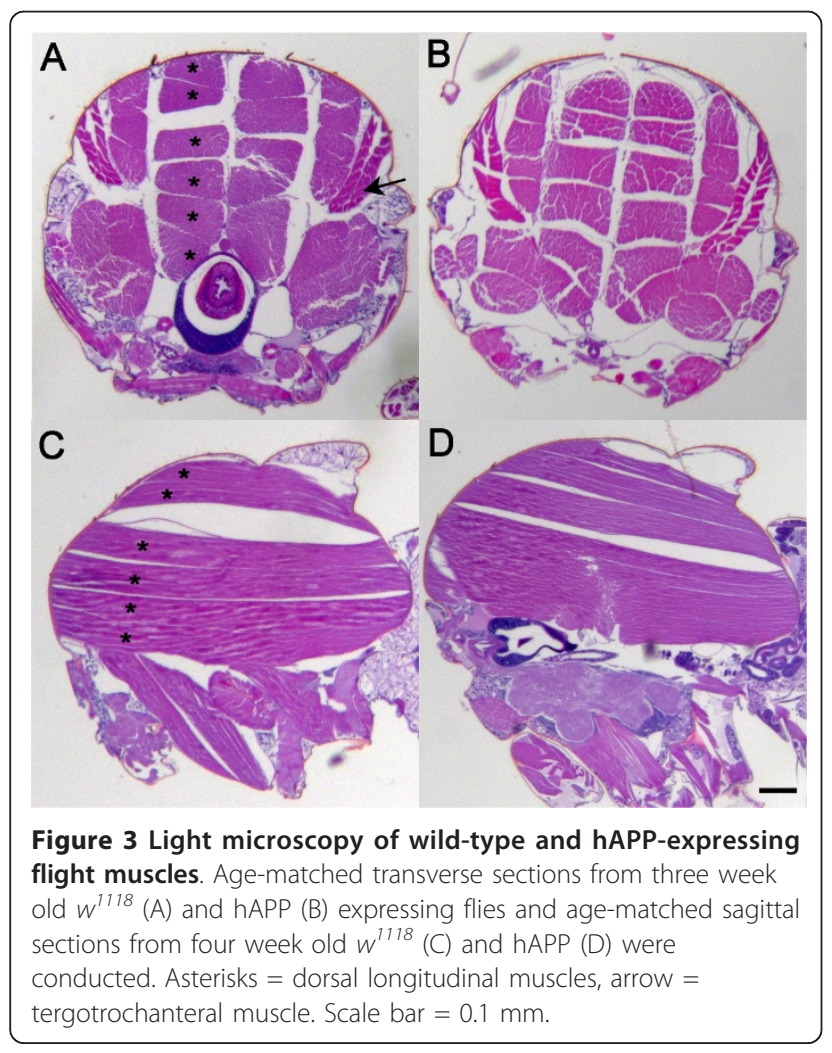

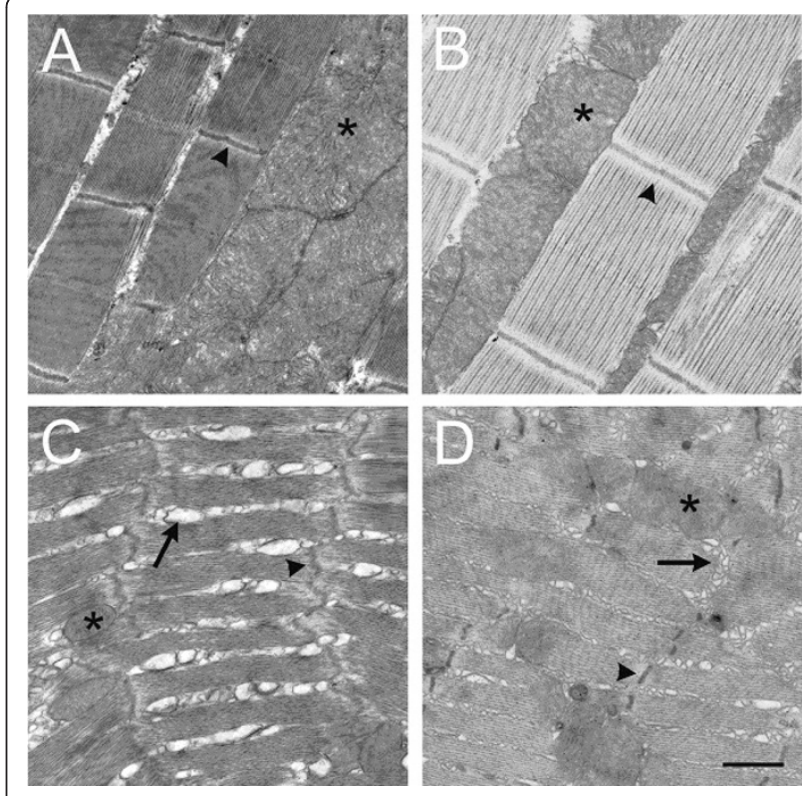

Figure 4 Transmission electron microscopy of wild-type and hAPP-expressing flight muscle. Dorsal longitudinal flight muscles (DLFM) from control $\left(w^{1118}, A\right)$ and hAPP (B) transgenic flies at three weeks of age. TTM muscles from control $\left(w^{1118}, C\right)$ and hAPP (D) transgenic animals at three weeks of age. Asterisks = mitochondria; Arrowheads $=$ Z bands; Arrows = sarcoplasmic reticulum. Scale bar $=1 \mu \mathrm{m}$. Note that variations in the plane of section contribute to apparent changes in the sizes of some ultrastructural components.

contractile elements perpendicular to the $\mathrm{Z}$ bands. At the subcellular level, the organization of the TTM muscles differs from that of the DLFM both in terms of sarcomeric structure and the abundance of internal membrane systems of the T-tubule and sarcoplasmic reticulum (SR) (Figure $4 \mathrm{C}$ and $4 \mathrm{D}$ ). At the electron microscopic level, 3 week old wild-type and hAPP transgenic TTM muscles were grossly indistinguishable (Figure $4 \mathrm{C}$ and $4 \mathrm{D})$.

\section{Environmental control of muscle pathogenesis in hAPP- expressing flies}

We performed many replicates of the climbing assay and consistently observed comparable age-dependent defects in transgenic animals. At one point in the study however, we switched from glass to polypropylene plastic vials for rearing the adults. While longevity was unchanged under these different rearing conditions (data not shown), the severity of the hAPP-induced climbing defect of flies cultured in the plastic vials was significantly reduced over the course of one month (Figure $5 \mathrm{~A}$ ). In order to verify that the rearing container impacted climbing behavior, cohorts of wild-type and hAPP-expressing animals were separated into glass or plastic vials and then subjected to the climbing assay. Wild-type animals displayed statistically improved 

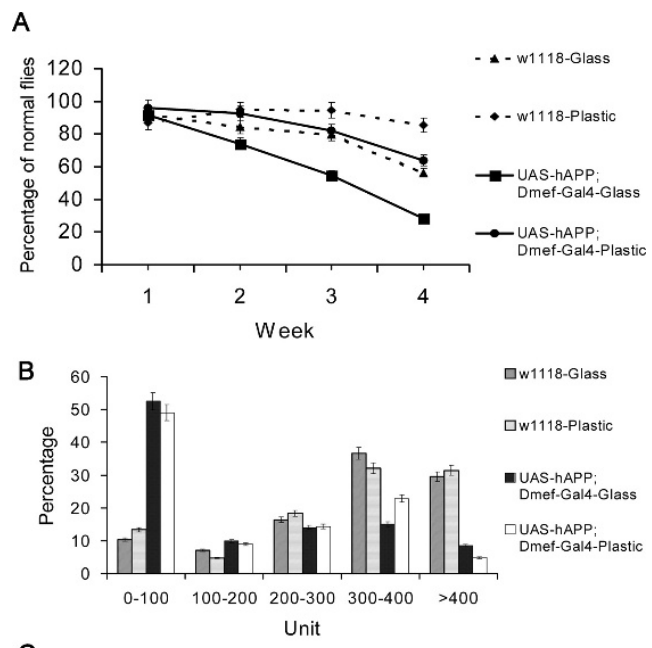

C

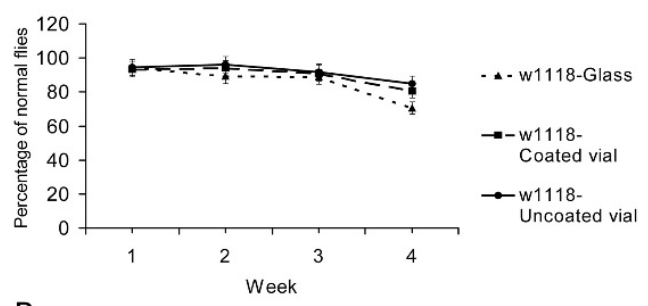

D

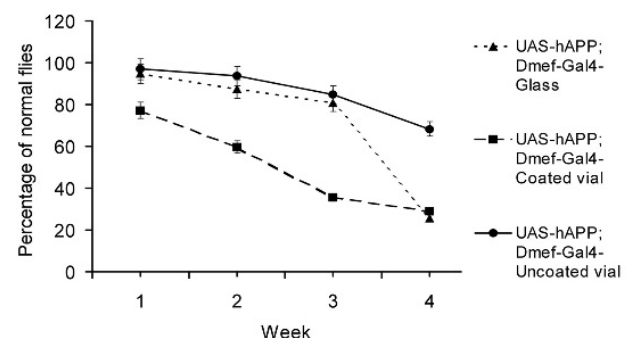

Figure 5 Effect of vial type on climbing behavior in wild-type and hAPP transgenic flies. A. The climbing ability of wild type and hAPP expressing flies reared in glass or plastic vials was determined. Percentage represents the portion of flies displaying normal behavior. $N=161$ and 171 for wild type in plastic and glass vials, 171 and 173 for transgenic flies in plastic and glass vials, respectively. Mean $+/$ - SEM. Climbing was statistically different by 4 weeks $(p<0.05)$. The error bars for the transgenic flies reared in glass vials were are not easily observed due to their small size. B. Flying assay was performed with 5 week old wild type flies cultured in glass (black) or plastic (white) vials and for hAPP expressing flies in glass (dark gray) or plastic vials (light gray). $\mathrm{N}=199$ and 126 for the wild-type animals reared in plastic and glass vials, and $N=126$ and 128 for transgenic flies in plastic and glass vials, respectively. Mean +/- SEM. C. Climbing assays were performed with wild type flies cultured in glass, uncoated or siliconized plastic vials. In this set of experiments the flies reared in glass vials displayed a slightly better performance relative to the animals represented in Figure 5A. $N=117,121$, and118, respectively. Mean +/- SEM. Climbing was statistically different by 4 weeks $(p<0.05) D$. Climbing assays were performed with hAPP expressing flies reared in glass, uncoated and siliconized plastic vials. $N=124,118$, and 141 , respectively. Mean + +- SEM. climbing activity in plastic vials $(\bullet)$ relative to glass vials (४) (85\% vs: $56 \%$ at week four; p < 0.05 ) (Figure 5A). A much more dramatic effect was observed for the hAPPexpressing animals. At 4 weeks $65 \%$ of plastic reared animals $(\bullet)$ could climb versus $25 \%$ for glass-reared animals $(-)(\mathrm{p}<0.05)$. The benefits of plastic versus glass vials were restricted to the leg muscles and did not extend to the flight muscles (Figure 5B), since we did not observe any differences in flying activity between these two populations. These data suggest that only muscles that are actively challenged are the ones affected by the type of rearing substrate.

One possible mechanism for the differential effects of the vial composition on the development of muscle weakness is that the smoothness of glass relative to plastic requires more muscle strength to climb. To test this hypothesis, we siliconized plastic vials to make their surfaces smoother, and then tested new groups of control and transgenic animals. Survival was comparable in all three types of vials (data not shown). Wild type control flies climbed equally well in all three types of vials until week 3 . After that, there was a small but statistically significant decline in climbing ability for flies reared in glass vials $(\boldsymbol{\bullet})$ relative to coated $(\boldsymbol{\bullet})$ and uncoated plastic vials $(\bullet)$ (Figure 5C). However, the effects of vial type were much more dramatic in the hAPP transgenic flies (Figure 5D). Transgenic flies reared in siliconized plastic vials (-) displayed a rapid loss of climbing activity beginning even after the first week relative to those reared in glass $(\mathbf{\Lambda})$ or uncoated plastic vials $(\bullet)$. Between three and four weeks, there was a dramatic decline in climbing for hAPP transgenic flies reared in glass vials. These data suggest that the environmental factors (vial material) can have a significant impact on APP-induced pathogenesis.

\section{Coexpression of human Parkin rescues a hAPP-mediated climbing defect}

Mutations in the ubiquitin E3 ligase Parkin is the primary cause of Autosomal Recessive Juvenile Parkinsonism [27]. Loss of Parkin function endangers some cells, most notably midbrain dopaminergic neurons. However, in vitro studies with muscle cells have demonstrated that expression of ectopic Parkin can protect muscle from the toxic effects of accumulation of amyloid peptides [28]. To determine if this protein could also protect skeletal muscles in vivo from hAPP-induced damage, we generated transgenic flies that express human Parkin and/or hAPP in the muscles, and then monitored the ability of the adults to climb (Figure 6). Flies expressing hAPP $(\mathbf{\Lambda})$ demonstrated a significant decline in climbing ability beginning in the third week. Co-expression of Parkin $(\checkmark)$ rescued this defect and allowed the flies to climb at wild type levels (Figure 6). 


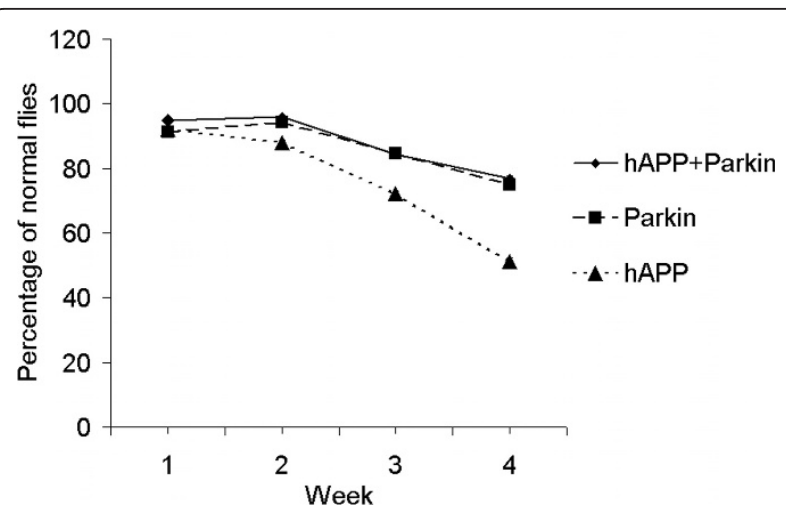

Figure 6 Ectopic Parkin rescues hAPP-induced climbing defects in transgenic flies. Climbing assays with transgenic flies expressing hAPP alone, hParkin alone, and hAPP plus hParkin in glass vials were conducted each week. $N=54$ for all genotypes. Mean +/- SEM.

APP has been the focus of intensive investigation for its possible role in human diseases, most notably Alzheimer's disease. Numerous studies have demonstrated that ectopic expression of APP, or its proteolytic products, most notably $A \beta_{42}$, can trigger synapse loss and neuron death in both in vitro and in vivo models $[9,10]$. In fly, ectopic expression of $A \beta_{42}$ or $A \beta_{40}$ results in age-dependent neuron loss [29]. Interestingly, males were more severely impacted than females, although this may reflect differences in driver expression rather than differential gender sensitivity.

These same proteins have been proposed to accumulate within aggregates in patients with other diseases as well, such as inclusion body myositis [30], although this observation has been controversial $[17,18]$. Nevertheless, experimental studies have demonstrated that ectopic expression of either APP or $A \beta_{42}$ is sufficient to induce muscle cell death both in vitro $[19,20]$ and in transgenic mouse models $[22,23,31]$. In the nematode Caenorhabditis elegans, expression of $\mathrm{A} \beta_{42}$ results in protein aggregations within body wall muscles that result in paralysis and reduced longevity [32-34].

The transgenic fly model described in the present study complements and extends some of the data obtained with mouse and worm models, as well as some of the features of s-IBM. In s-IBM patients and animal models designed to simulate the disorder, individuals produce muscles that appear to be morphologically and physiologically normal, but develop progressive agedependent muscle weakness in mid-to-late adulthood $[12,22,32]$ (Figure 1C and 1D). This loss of muscle strength is not accompanied by detectable changes in neuromuscular synapse activity, suggesting that the defects arise within the muscles themselves. Indeed, we did not observe any changes in following frequency or spike amplitude in the muscles of our hAPP-expressing animals (Figure 2). These data would argue against a negative influence of hAPP expression in the small subset of circadian neurons in the brain that also express MEF2 [26]

The fly model described in this study does differ from both s-IBM and other hAPP transgenic mouse and worm models in that they did not produce protein aggregates within the sarcoplasm that can be detected with either Congo Red staining (data not shown) or ultrastructural analysis (Figure 4). It is possible that the level of hAPP expression was below the threshold required for macro-aggregation, or alternatively, that flies have intracellular mechanisms that limit aggregate formation. It has been noted that flies do not make inclusion bodies in the skeletal muscles with other aggregation prone proteins, like the polyglutamine-rich Huntington protein [35]. Nevertheless, the present fly model may provide insight into the molecular mechanisms that mediate hAPP induced pathology.

Several mechanisms have been proposed for the toxic effects of APP on skeletal muscle function including: defective regulation of ryanodine receptor-dependent sarcoplasmic $\mathrm{Ca}^{2+}$ release [36], CD8+ cytotoxic T cell invasion [37], an autophagic mechanism [38], and myostatin activity [39].

The observation that ectopic expression of the ubiquitin E3 ligase Parkin can rescue the hAPP-associated defects in climbing in our transgenic model agrees well with in vitro results from other groups $[16,27,40,41]$. At least two possible Parkin associated mechanisms can ameliorate ectopic APP-induced behavioral defects. In mammalian muscle, Parkin prevents hAPP-induced muscle degeneration by inhibiting accumulation of toxic $A \beta s$ and also protects cells from mitochondrial-specific toxins like rotenone and carbonyl cyanide 3-chlorophenylhydrazone, but not from other toxins like calcium ionophore $\mathrm{A} 23187$ or $\mathrm{H}_{2} \mathrm{O}_{2}$ [28]. Loss of function mutations in the parkin gene result in age-dependent defects in flight muscle maintenance in fly and are also associated with mitochondrial defects [42]. Interestingly, these defects can be prevented by increased mitochondrial fission [43]. Taken together, these data support the hypothesis that Parkin may reduce mitochondrial oxidative stress or maintain respiratory functions to help preserve mitochondrial integrity.

We have demonstrated that environmental factors, such as rearing surface (glass versus plastic), have a dramatic effect on the timing and severity of hAPP-induced pathogenesis, suggesting a possible interplay between environmental and genetic factors. To our knowledge, this is the first report of any behavioral activities that can be tied to the material used for rearing flies. The simple manipulation of changing the rearing vessel may represent a valuable tool for genetic screens in fly designed to identify work-associated genes in fly muscle. 
Only limited data exist on the role of exercise in the progression of myopathies in human [44,45]. Interestingly, Arnardottir et al. [44] have suggested that moderate exercise may retard the symptoms of s-IBM. Rearing flies in plastic vials greatly reduced the timing and severity of symptoms relative to rearing in glass (Figure $5 \mathrm{~A})$. Since the only difference between animals reared in glass versus plastic was the nature of the vessel, we speculated that surface properties accounted for the observed effects. Glass vials have a smoother surface than plastic and therefore it is presumably harder for the fly to climb. This extra work would increase the mechanical stress on the muscle fibers and might contribute to damage or generation of reactive oxygen species (ROS) by mitochondria. We tried to test this hypothesis directly by setting up devices that would force the animal to walk more, such as placing the vials on a slowly moving rocking table. Unfortunately, these efforts to voluntarily increase motor activity did not appear to alter animal behavior, so instead we changed the surface properties of the plastic vials by siliconizing them. This subtle manipulation had a profound effect on the time course and severity of the hAPP-induced abnormal activities. This effect was specific to the muscles that were forced to work, since this treatment did not alter flying behavior.

\section{Conclusions}

In this study, we generate a transgenic Drosophila model that targets the expression of hAPP to the skeletal muscles in order to understand its effect on muscle development and degeneration in the adult. Ectopic expression of hAPP causes age- and activity-dependent muscle weakness without concomitant structural damage that can be discerned at either the light or electron microscopic levels. The effects of ectopic hAPP can be ameliorated by hParkin expression. Taken together, these data suggest that in Drosophila, hAPP-induced muscle deficits are mediated by not only genetic, but also environmental factors, and that muscle work/stress may contribute to pathogenesis. This animal model is useful to identify additional genetic and environmental modulators and understand underlying mechanisms.

\section{Methods \\ Generation of transgenic UAS-human Parkin Drosophila lines}

In order to generate UAS-human Parkin, pRK5-mycParkin generously provided by Dr. Dawson [46] and a pUAST vector were digested with EcoRI and NotI restriction enzymes (Promega) and ligated at $16^{\circ} \mathrm{C}$ overnight with T4 ligase (Promega). The construct, pUASTmyc-human Parkin was sent to the Transgenic Drosophila Fly Core (Charlestown, MA) for embryonic injections. Transgenic lines were selected and cultured at room temperature.

\section{Drosophila}

Transgenic flies expressing human APP under the control of the Gal4 upstream activating sequence (UAS) were provided by Dr. Kalpana White [24]. A DmefGAL4 line driving the expression Gal4 in mesodermal precursors [25] was provided by Dr. Leo Pallanck. Progenies from these crosses were maintained at $25^{\circ} \mathrm{C}$ with 12 hour-light/dark cycle. Both males and females were used in each experiment.

\section{Climbing Assay and flight assay}

Flies were divided into 5-7 groups of 25 flies each and reared in standard glass or plastic vials (Genesee Scientific) as noted. In some experiments plastic vials were siliconized with glass cleaner (Rain-X). The vials were exchanged every two to three days and animals were scored weekly in the climbing assay. Briefly, after a 1 minute acclimation, the percentage of flies that could climb past the $30 \mathrm{ml}$ mark in a $50 \mathrm{ml}$ cylinder within 30 seconds was scored as successful. Trials were performed 3 times/vial. After five weeks, the flies were subjected to the flying test. The animals were dropped into a $500 \mathrm{ml}$ graduate cylinder coated with mineral oil. The number of immobilized flies was determined for each $100 \mathrm{ml}$ section of the cylinder.

\section{Statistical Analysis}

Statistical significance was determined using Student's T test and one-way-analysis of variances (ANOVA) using GraphPad Prism software (GraphPad Software, La Jolla, CA). Data were considered statistically significant when their $\mathrm{p}$ values were 0.05 or less.

\section{Electrophysiology}

Standard methods were used to get intracellular recordings from the dorsal longitudinal flight muscle (DLFM) and the tergotrochanteral muscle (TTM) [47]. Flies were anesthetized and waxed, ventral side down, onto a small podium in a Petri dish. The motorneurons were either indirectly stimulated via the Giant Fiber (GF) in the brain or directly with thoracic stimulation using two etched tungsten electrodes and by providing pulses of 40-60V for $0.03 \mathrm{~ms}$ using a Grass S44 stimulator (Grass Instruments). A tungsten electrode placed in the abdominal cavity served as a ground. Glass electrodes pulled to a resistance of 40-60MW were filled with saline and were driven through the cuticle into the DLFM and TTM muscle fibers. Signals were amplified, digitized and analyzed with pClamp 10 software to monitor amplitude size and the ability of the muscle membrane to follow multiple stimuli at 100HZ (Getting Instruments and Molecular Devices). 


\section{Microscopy}

Flies were collected at different ages, the heads and abdomens removed, and the carcass immersed in $4 \%$ paraformaldehyde overnight at $4{ }^{\circ} \mathrm{C}$. They were then dehydrated, embedded in paraffin and sectioned at 10 $\mu \mathrm{m}$ followed by staining with hematoxylin and eosin for light microscopy. For transmission electron microscopy, flies were prepared as previously described [48,49]. A fly was placed thorax facing upward and fully covered in a droplet of Optimal Cutting Temperature (OCT) compound on a glass microscope slide. The fly was snap frozen in liquid nitrogen $\left(-196^{\circ} \mathrm{C}\right)$ and then gently fractured with a liquid nitrogen-chilled razor blade struck with a hammer to longitudinally bisect the flies. Care was taken to ensure that the droplet of tissue was completely submerged in liquid nitrogen throughout the fracturing process, so that at no time was any sample exposed to air and that the razor blade contacted the OCT compound, not the thorax itself. The split pieces were immediately transferred to a container of primary fixative made of $2.5 \%$ glutaraldehyde in $0.1 \mathrm{M}$ phosphate buffer at $\mathrm{pH} 7.4$, and allowed to thaw and fix overnight at $4^{\circ} \mathrm{C}$. The fixative was changed several times to ensure its concentration was not altered by the thawing OCT. After rinsing in $0.1 \mathrm{M} \mathrm{NaPO}_{4}$ buffer at $\mathrm{pH} \mathrm{7.4,} \mathrm{the} \mathrm{tis-}$ sue was post-fixed in $2 \%$ osmium tetroxide in $0.1 \mathrm{M}$ $\mathrm{NaPO}_{4}$ buffer at $\mathrm{pH} 7.4$ for one hour. The sample was then rinsed in distilled water and stained with $1 \%$ uranyl acetate for 30 minutes. It was then rinsed in water and dehydrated through an ethanol series from 30\% to $100 \%$. The sample was then infiltrated with Spurr's extra low viscosity resin, and after embedding, mounted fracture-surface up and thin-sectioned with a diamond knife for TEM analysis. Multiple sections from at least eight different flies of each genotype and age were examined for each experiment.

\section{List of Abreviations}

hAPP: human amyloid precursor protein; AD: Azheimer's disease; s-IBM: sporadic inclusion body myositis; A $\beta$ : $\beta$ amyloid peptide; UAS: upstream activating sequence; LacZ: $\beta$-galactosidase; Dmef: Drosophila myocyte enhancer factor; GF: Giant Fiber; DLFM: dorsal longitudinal flight muscle; TTMn: tergotrochanteral motorneuron; TTM: tergotrochanteral muscle; SR: sarcoplasmic reticulum; ROS: reactive oxygen species.

\footnotetext{
Acknowledgements

We thank Dr. by Dr. Kalpana White for the UAS-hAPP transgenic flies and Dr. Leo Pallanck for the Dmef-GAL4 line. This project was supported by NIH grant R01NS042898 to LMS. MR, SS., and CTW were supported by Howard Hughes Medical Institute Grant 52006307 to Mount Holyoke College and TAG was supported by a grant R01HD050725 from the NIH. We thank Ms. Christine Brown for technical and editorial assistance.
}

\section{Author details}

${ }^{1}$ Molecular and Cellular Biology Program, University of Massachusetts, Amherst, MA 01003, USA. ²Department of Biology, University of Massachusetts, Amherst, MA 01003, USA. ㄹDepartment of Biological Sciences, Mount Holyoke College, South Hadley, MA 01075, USA. ${ }^{4}$ Department of Biological Sciences, Florida Atlantic University, Boca Raton, FL 33431, USA. ${ }^{5}$ Pioneer Valley Life Sciences Institute, 3601 Main Street, Springfield, MA, 01199, USA.

\section{Authors' contributions}

CK generated the transgenic animals, performed most of the behavioral assays and analyses, and co-wrote the manuscript. SS performed some behavioral assays as part of her honors thesis work. MR and SS prepared the samples and conducted transmission electron microscopic analysis (as part of SS's honors thesis). TG performed the electrophysiological studies. BB performed the light microscopy studies. SR and CTW performed the analyses of the hParkin expressing flies. CTW and LS designed, discussed and helped co-write the manuscript with CK. All authors read and approved the final manuscript.

\section{Competing interests}

The authors declare that they have no competing interests.

Received: 30 September 2010 Accepted: 25 April 2011

Published: 25 April 2011

\section{References}

1. Thinakaran G, Koo EH: Amyloid precursor protein trafficking, processing, and function. J Biol Chem 2008, 283:29615-29619.

2. Meziane H, Dodart JC, Mathis C, Little S, Clemens J, Paul SM, Ungerer A: Memory-enhancing effects of secreted forms of the beta-amyloid precursor protein in normal and amnestic mice. Proc Natl Acad Sci USA 1998, 95:12683-12688.

3. Dodart JC, Mathis C, Ungerer A: The beta-amyloid precursor protein and its derivatives: from biology to learning and memory processes. Rev Neurosci 2000, 11:75-93.

4. Citron M, Oltersdorf T, Haass C, McConlogue L, Hung AY, Seubert P, VigoPelfrey C, Lieberburg I, Selkoe DJ: Mutation of the beta-amyloid precursor protein in familial Alzheimer's disease increases beta-protein production. Nature 1992, 360:672-674.

5. Folin M, Baiguera S, Conconi MT, Pati T, Grandi C, Parnigotto PP, Nussdorfer GG: The impact of risk factors of Alzheimer's disease in the Down syndrome. Int J Mol Med 2003, 11:267-270.

6. Felsenstein KM, Hunihan LW, Roberts SB: Altered cleavage and secretion of a recombinant beta-APP bearing the Swedish familial Alzheimer's disease mutation. Nat Genet 1994, 6:251-255.

7. Haass C, Selkoe D: Soluble protein oligomers in neurodegeneration: lessons from the Alzheimer's amyloid beta-peptide. Nat Rev Mol Cell Biol 2007, 8:101-112.

8. Iwatsubo T, Odaka A, Suzuki N, Mizusawa $H$, Nukina $N$, Ihara $Y$ : Visualization of $A b 42(43)$ and $A b 40$ in senile plaques with end-specific $A b$ monoclonals: Evidence that aninitially deposited species is Ab42(43). Neuron 1994, 13:45-53.

9. Puttfarcken PS, Manelli AM, Neilly J, Frail DE: Inhibition of age-induced beta-amyloid neurotoxicity in rat hippocampal cells. Exp Neurol 1996, 138:73-81.

10. Johnson-Wood K, Lee M, Motter R, Hu K, Gordon G, Barbour R, Khan K, Gordon M, Tan H, Games D, Lieberburg I, Schenk D, Seubert P, McConlogue L: Amyloid precursor protein processing and A beta42 deposition in a transgenic mouse model of Alzheimer disease. Proc Natl Acad Sci USA 1997, 94:1550-1555.

11. Askanas $V$, Engel WK: Unfolding story of inclusion-body myositis and myopathies: role of misfolded proteins, amyloid-beta, cholesterol, and aging. J Child Neurol 2003, 18:185-190.

12. Amato AA, Barohn RJ: Inclusion body myositis: old and new concepts. J Neurol Neurosurg Psychiatry 2009, 80:1186-1193.

13. Dalakas MC: Immunotherapy of myositis: issues, concerns and future prospects. Nat Rev Rheumatol 2010, 6:129-137.

14. Vattemi G, Nogalska A, King Engel W, D'Agostino C, Checler F, Askanas V: Amyloid-beta42 is preferentially accumulated in muscle fibers of 
patients with sporadic inclusion-body myositis. Acta Neuropathol 2009, 117:569-574.

15. Sherriff FE, Joachim CL, Squier MV, Esiri MM: Ubiquitinated inclusions in inclusion-body myositis patients are immunoreactive for cathepsin $D$ but not beta-amyloid. Neurosci Lett 1995, 194:37-40.

16. Parker KC, Kong SW, Walsh RJ, Bch, Salajegheh M, Moghadaszadeh B, Amato AA, Nazareno R, Lin YY, Krastins B, Sarracino DA, Beggs AH, Pinkus JL, Greenberg SA: Fast-twitch sarcomeric and glycolytic enzyme protein loss in inclusion body myositis. Muscle Nerve 2009, 39:739-753.

17. Greenberg SA: How citation distortions create unfounded authority: analysis of a citation network. BMJ 2009, 339:b2680.

18. Greenberg SA: Inclusion body myositis: review of recent literature. Curr Neurol Neurosci Rep 2009, 9:83-89.

19. Askanas V, McFerrin J, Baqué S, Alvarez RB, Sarkozi E, Engel WK: Transfer of beta-amyloid precursor protein gene using adenovirus vector causes mitochondrial abnormalities in cultured normal human muscle. Proc Nat Acad Sci USA 1996, 93:1314-1319.

20. Querfurth HW, Suhara T, Rosen KM, McPhie DL, Fujio Y, Tejada G, Neve RL, Adelman LS, Walsh K: Beta-amyloid peptide expression is sufficient for myotube death: implications for human inclusion body myopathy. Mol Cell Neurosci 2001, 17:793-810.

21. Moussa CE, Fu Q, Kumar P, Shtifman A, Lopez JR, Allen PD, LaFerla F, Weinberg D, Magrane J, Aprahamian T, Walsh K, Rosen KM, Querfurth HW: Transgenic expression of beta-APP in fast-twitch skeletal muscles leads to calcium dyshomeostasis and IBM-like pathology. FASEB J 2006, 20:2165-2167.

22. Sugarman MC, Kitazawa M, Baker M, Caiozzo VJ, Querfurth HW, LaFerla FM: Pathogenic accumulation of APP in fast twitch muscle of IBM patients and a transgenic model. Neurobiol Aging 2006, 27:423-432.

23. Sugarman MC, Yamasaki TR, Oddo S, Echegoyen JC, Murphy MP, Golde TE, Jannatipour M, Leissring MA, LaFerla FM: Inclusion body myositis-like phenotype induced by transgenic overexpression of beta APP in skeletal muscle. Proc Natl Acad Sci USA 2002, 99:6334-6339.

24. Luo L, Tully T, White K: Human amyloid precursor protein ameliorates behavioral deficit of flies deleted for Appl gene. Neuron 1992, 9:595-605.

25. Lilly B, Galewsky S, Firulli AB, Schulz RA, Olson EN: D-MEF2: a MADS box transcription factor expressed in differentiating mesoderm and muscle cell lineages during Drosophila embryogenesis. Proc Natl Acad Sci USA 1994, 91:5662-5666.

26. Blanchard FJ, Collins B, Cyran SA, Hancock DH, Taylor MV, Blau J: The transcription factor Mef2 is required for normal circadian behavior in Drosophila. J Neurosci 2010, 30:58555865.

27. Kitada T, Asakawa S, Hattori N, Matsumine H, Yamamura Y, Minoshima S, Yokochi M, Mizuno Y, Shimizu N: Mutations in the parkin gene cause autosomal recessive juvenile parkinsonism. Nature 1998, 392:605-608.

28. Rosen KM, Veereshwarayya V, Moussa CE, Fu Q, Goldberg MS, Schlossmacher MG, Shen J, Querfurth HW: Parkin protects against mitochondrial toxins and beta-amyloid accumulation in skeletal muscle cells. J Biol Chem 2006, 281:12809-12816.

29. lijima K, Liu HP, Chiang AS, Hearn SA, Konsolaki M, Zhong Y: Dissecting the pathological effects of human Abeta 40 and Abeta42 in Drosophila: a potential model for Alzheimer's disease. Proc Natl Acad Sci USA 2004, 101:6623-6628.

30. Askanas V, Alvarez RB, Engel WK: beta-Amyloid precursor epitopes in muscle fibers of inclusion body myositis. Ann Neurol 1993, 34:551-60.

31. Turdi S, Guo R, Huff AF, Wolf EM, Culver B, Ren J: Cardiomyocyte contractile dysfunction in the APPswe/PS1dE9 mouse model of Alzheimer's disease. PLoS One 2009, 24:e6033.

32. Link CD: Expression of human beta-amyloid peptide in transgenic Caenorhabditis elegans. Proc Natl Acad Sci USA 1995, 92:9368-9372.

33. Drake J, Link CD, Butterfield DA: Oxidative stress precedes fibrillar deposition of Alzheimer's disease amyloid beta-peptide (1-42) in a transgenic Caenorhabditis elegans model. Neurobiol Aging 2003, 24:415-420.

34. Minniti AN, Rebolledo DL, Grez PM, Fadic R, Aldunate R, Volitakis I, Cherny RA, Opazo C, Masters C, Bush Al, Inestrosa NC: Intracellular amyloid formation in muscle cells of Abeta-transgenic Caenorhabditis elegans: determinants and physiological role in copper detoxification. Mol Neurodegener 2009, 4:2.

35. Ravikumar B, Vacher C, Berger Z, Davies JE, Luo S, Oroz LG, Scaravilli F, Easton DF, Duden R, O'Kane CJ, Rubinsztein DC: Inhibition of mTOR induces autophagy and reduces toxicity of polyglutamine expansions in fly and mouse models of Huntington disease. Nat Genet 2004, 36:585-595.

36. Shtifman A, Ward CW, Laver DR, Bannister ML, Lopez JR, Kitazawa M, Laferla FM, Ikemoto N, Querfurth HW: Amyloid-beta protein impairs Ca(2 +) release and contractility in skeletal muscle. Neurobiol Aging 2010, 31:2080-2090.

37. Kitazawa M, Trinh DN, LaFerla FM: Inflammation induces tau pathology in inclusion body myositis model via glycogen synthase kinase-3beta. Ann Neurol 2008, 64:15-24.

38. Lünemann JD, Schmidt J, Dalakas MC, Münz C: Macroautophagy as a pathomechanism in sporadic inclusion body myositis. Autophagy 2007, 3:384-386

39. Wójcik S, Engel WK, McFerrin J, Askanas V: Myostatin is increased and complexes with amyloid-beta within sporadic inclusion-body myositis muscle fibers. Acta Neuropathol 2005, 110:173-177.

40. Paciello O, Wójcik S, Engel WK, McFerrin J, Askanas V: Parkin and its association with alpha-synuclein and AbetaPP in inclusion-body myositis and AbetaPP-overexpressing cultured human muscle fibers. Acta Myol 2006, 25:13-22.

41. Rosen KM, Moussa CE, Lee HK, Kumar P, Kitada T, Qin G, Fu Q, Querfurth HW: Parkin reverses intracellular beta-amyloid accumulation and its negative effects on proteasome function. J Neurosci Res 2010, 88:167-178.

42. Greene JC, Whitworth AJ, Kuo I, Andrews LA, Feany MB, Pallanck LJ: Mitochondrial pathology and apoptotic muscle degeneration in Drosophila parkin mutants. Proc Natl Acad Sci USA 2003, 100:4078-4083.

43. Park J, Lee G, Chung J: The PINK1-Parkin pathway is involved in the regulation of mitochondrial remodeling process. Biochem Biophys Res Commun 2009, 378:518-523.

44. Arnardottir S, Alexanderson H, Lundberg IE, Borg K: Sporadic inclusion body myositis: pilot study on the effects of a home exercise program on muscle function, histopathology and inflammatory reaction. J Rehabil Med 2003, 35:31-35.

45. Alexanderson $\mathrm{H}$, Lundberg IE: The role of exercise in the rehabilitation of idiopathic inflammatory myopathies. Curr Opin Rheumatol 2005, 17:164-171.

46. Zhang Y, Gao J, Chung KK, Huang H, Dawson VL, Dawson TM: Parkin functions as an E2-dependent ubiquitin- protein ligase and promotes the degradation of the synaptic vesicle-associated protein, CDCrel-1. Proc Natl Acad Sci USA 2000, 97:13354-13359.

47. Allen MJ, Godenschwege TA: Electrophysiological Recordings from the Giant Fiber System. Cold Spring Harb Protoc 2010, 8, (2010) pdb.prot5453.

48. Chartier A, Benoit B, Simonelig M: A Drosophila model of oculopharyngeal muscular dystrophy reveals intrinsic toxicity of PABPN1. EMBO J 2006, 25:2253-2262.

49. Allikian MJ, Bhabha G, Dospoy P, Heydemann A, Ryder P, Earley JU, Wolf MJ, Rockman HA, McNally EM: Reduced life span with heart and muscle dysfunction in Drosophila sarcoglycan mutants. Hum Mol Genet 2007, 16:2933-2943.

doi:10.1186/1472-6793-11-7

Cite this article as: Kim et al:: Expression of human amyloid precursor protein in the skeletal muscles of Drosophila results in age- and activity-dependent muscle weakness. BMC Physiology 2011 11:7.

\section{Submit your next manuscript to BioMed Central and take full advantage of:}

- Convenient online submission

- Thorough peer review

- No space constraints or color figure charges

- Immediate publication on acceptance

- Inclusion in PubMed, CAS, Scopus and Google Scholar

- Research which is freely available for redistribution 